\title{
Development of a Polarized 3He++ Ion Source for the EIC
}

\section{Musgrave*广and R. Milner}

Laboratory for Nuclear Science, Massachusetts Institute of Technology, Cambridge, MA, USA

E-mail: *musgrave@mit.edu

\author{
G. Atoian, E. Beebe, S. Ikeda, S. Kondrashev, M. Okamura, A. Poblaguev, D. \\ Raparia, J. Ritter, S. Trabocchi, and A. Zelenski \\ Brookhaven National Laboratory, Upton, NY, USA
}

\section{J. Maxwell}

Thomas Jefferson National Accelerator Facility, Newport New, VA, USA

\begin{abstract}
The capability of accelerating a high-intensity polarized ${ }^{3} \mathrm{He}$ ion beam would provide an effective polarized neutron beam for new high-energy QCD studies of nucleon structure. This development is essential for the future Electron Ion Collider, which could use a polarized ${ }^{3} \mathrm{He}$ ion beam to probe the spin structure of the neutron. The proposed polarized ${ }^{3} \mathrm{He}$ ion source is based on the Electron Beam Ion Source (EBIS) currently in operation at Brookhaven National Laboratory. ${ }^{3} \mathrm{He}$ gas would be polarized within the $5 \mathrm{~T}$ field of the EBIS solenoid via Metastability Exchange Optical Pumping (MEOP) and then pulsed into the EBIS vacuum and drift tube system where the ${ }^{3} \mathrm{He}$ will be ionized by the $10 \mathrm{Amp}$ electron beam. The goal of the polarized ${ }^{3} \mathrm{He}$ ion source is to achieve $2.5 \times 10^{11}{ }^{3} \mathrm{He}^{++} /$pulse at $70 \%$ polarization. An upgrade of the EBIS is currently underway. An absolute polarimeter and spin-rotator is being developed to measure the ${ }^{3} \mathrm{He}$ ion polarization at $6 \mathrm{MeV}$ after initial acceleration out of the EBIS. The source is being developed through collaboration between BNL and MIT.
\end{abstract}

The 18th International Workshop on Polarized Sources, Targets, and Polarimetry, PSTP2019 23-27 September, 2019

Knoxville, Tennessee

\footnotetext{
* Speaker.

${ }^{\dagger}$ For the BNL-MIT Polarized ${ }^{3} \mathrm{He}$ Ion Source Collaboration
} 


\section{Introduction}

The Electron Ion Collider (EIC) will enable the study of the spin structure of protons and neutrons with unprecedented precision. However, this statistical precision requires high intensity hadron beams with high polarization. RHIC has the capability to create polarized proton beams, but the study of polarized neutrons requires a suitable proxy for use in an accelerator. The ${ }^{3} \mathrm{He}$ nucleus has an $88.6 \%$ probability of being in its spatially symmetric S-state, where the protons form a singlet and the neutron carries the nuclear spin, which makes it a viable surrogate for a polarized neutron beam. However, an EIC will require a much higher intensity and polarization than achieved by previously developed polarized ${ }^{3} \mathrm{He}$ ion sources [1, 2, 3]. For this reason, development of a polarized ${ }^{3} \mathrm{He}$ ion beam has been identified as an R\&D priority for the EIC [4, 5, 6], and the BNLMIT polarized ${ }^{3} \mathrm{He}$ ion source collaboration has pursued this R\&D [7, 8, 9, 10, 11, 12, 13]. The design goal of the polarized ${ }^{3} \mathrm{He}$ ion source is $2.5 \times 10^{113} \mathrm{He}^{++} / \mathrm{s}$ with $70 \%$ polarization in a $20 \mu \mathrm{s}$ pulse, which equates to a peak current of $\approx 4 \mathrm{~mA}$.

\section{2. ${ }^{3}$ He Polarization at 5 Tesla}

Before ionization, ${ }^{3} \mathrm{He}$ gas at $1-5 \mathrm{mbar}$ is polarized in a cylindrical glass polarization cell within a $5 \mathrm{~T}$ magnetic field with the technique of metastability exchange optical pumping (MEOP) $[14,15]$. During MEOP, an RF excitation is induced in the ${ }^{3} \mathrm{He}$ gas to populate the $2^{3} \mathrm{~S}$ metastable state, which is optically pumped by circularly polarized $1083 \mathrm{~nm}$ light, and the nuclear spin state is simultaneously polarized by hyperfine coupling. A ${ }^{3} \mathrm{He}$ cell with an RF discharge can be seen in Fig. 1a, and Fig. 1b shows how the RF discharge is localized around the parameter of the ${ }^{3} \mathrm{He}$ polarization cell in high magnetic fields. The relatively new technique of high-field MEOP is similar with the additional complication that the spectra lines of the ${ }^{3} \mathrm{He}$ are Zeeman shifted by the $5 \mathrm{~T}$ field, as can be seen in Fig. 2. The pumping laser used to polarized the ${ }^{3} \mathrm{He}$ gas is a $5 \mathrm{~W}$ Lumibird fiber laser. The laser's central frequency is targeted at the $\mathrm{C} 0$ resonance line of ${ }^{3} \mathrm{He}$ $276769.46 \mathrm{GHz}$ with a $300 \mathrm{GHz}$ thermal tuning range, so that all four of the Zeeman shifted pump lines at $5 \mathrm{~T}$ can be reached. The laser also has a $2 \mathrm{GHz}$ linewidth, which is matched to the thermal Doppler broadening of the resonance lines. The ${ }^{3} \mathrm{He}$ gas polarization is measured by monitoring the transmission spectrum of a weak probe beam from a $1083 \mathrm{~nm}$ Toptica diode laser at either of the pair small spectral peaks shown in Fig. 2 at $-125 \mathrm{GHz}$ or $155 \mathrm{GHz}$.

We are developing an open cell ${ }^{3} \mathrm{He}$ polarizing system complete with ${ }^{3} \mathrm{He}$ gas purification. A ${ }^{3} \mathrm{He}$ cryo-purification system built from a modified cryopump effectively removes contaminants from the ${ }^{3} \mathrm{He}$ gas such that at a temperature of $46 \mathrm{~K}$ the cryo-purifier completely eliminates hydrogen, water, hydrocarbons, and argon from the gas spectrum. Additionally, adjusting the cyropurifier temperature enables fine control of the ${ }^{3} \mathrm{He}$ pressure. This cryo-purifier will allow routine operation of a flowing system to provide a continuous polarized ${ }^{3} \mathrm{He}^{++}$ion beam to the EIC. The open ${ }^{3} \mathrm{He}$ polarization cell has a relaxation time of about $30 \mathrm{~s}$, which is limited by the metal surfaces of valves and gas contamination. Tests with polarization cells attached to the gas handling system and cryo-purifer in a $3 \mathrm{~T}$ field have consistently achieved polarizations greater than $80 \%$ in the 1-3 mbar range. 


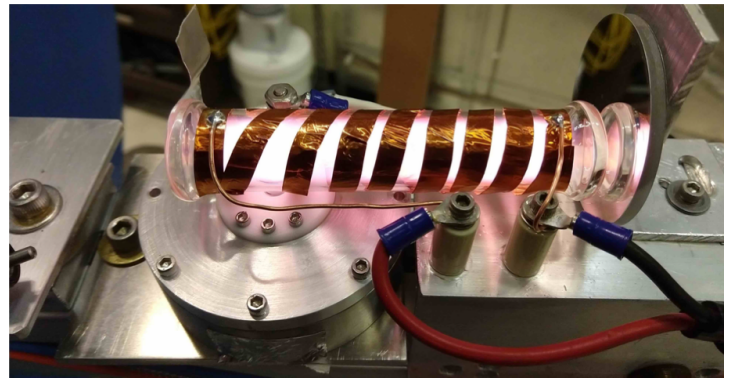

(a) ${ }^{3} \mathrm{He}$ polarization cell.

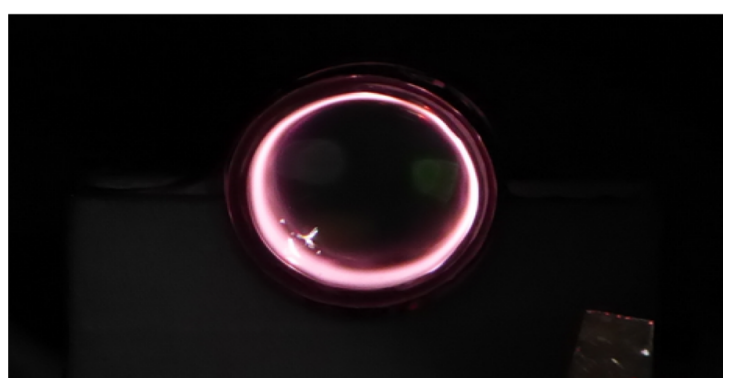

(b) RF discharge in $5 \mathrm{~T}$.

Figure 1: Images of ${ }^{3} \mathrm{He}$ polarization cells with an $\mathrm{RF}$ discharge in a $5 \mathrm{~T}$ magnetic field.

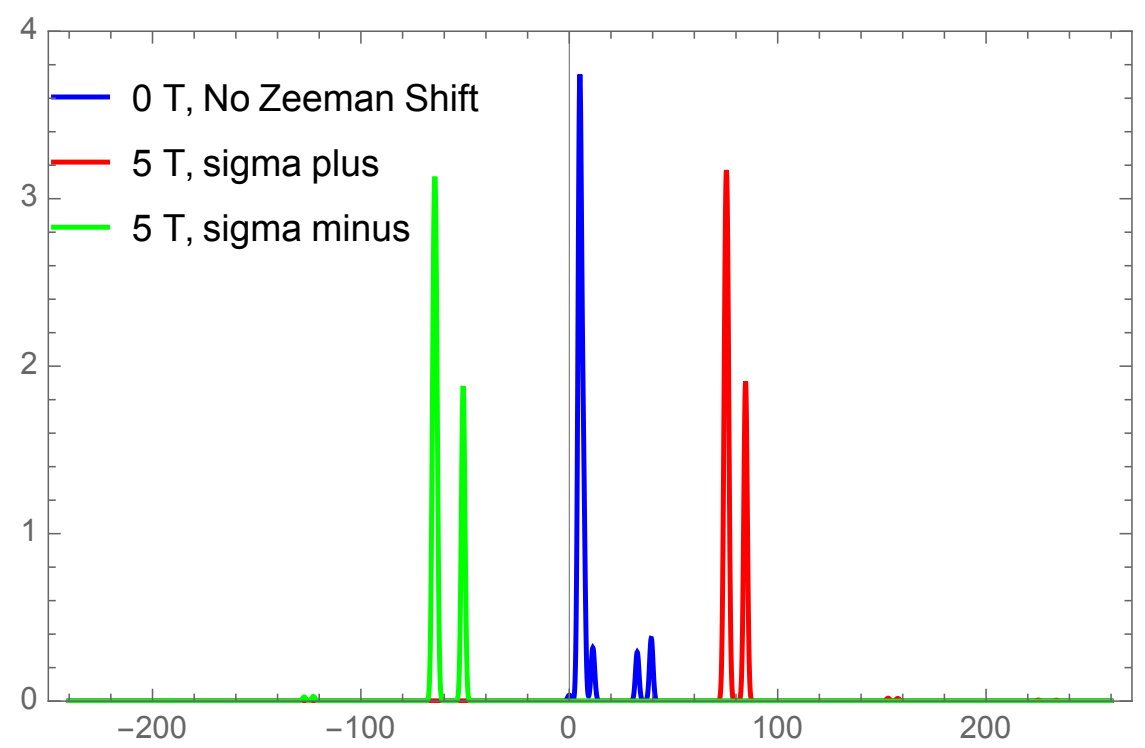

Figure 2: The Zeeman shifted spectra of ${ }^{3} \mathrm{He}$ relative to the $\mathrm{C} 1$ frequency at $0 \mathrm{~T}$ in units of $\mathrm{GHz}$. The four large red and green peaks can all be used to optically pump ${ }^{3} \mathrm{He}$. The small resonance peaks at $-125 \mathrm{GHz}$ and $155 \mathrm{GHz}$ are used with the probe laser polarimeter.

\section{RHIC EBIS \& Extended EBIS Upgrade}

The Electron Beam Ion Source (EBIS) is the primary source of charged ions from D to U for the Relativistic Heavy Ion Collider (RHIC) at Brookhaven National Lab [16]. The EBIS is currently being upgraded to enable ion production from gas injection and to increase the ion trap capacity. The primary purpose of the gas injection system is for the injection of polarized ${ }^{3} \mathrm{He}$ gas to produce ${ }^{3} \mathrm{He}^{++}$ions. The EBIS consists of a $10 \mathrm{~A}$ electron beam that is compressed by a $5 \mathrm{~T}$ solenoidal magnetic field. Low charge state ions are injected into the EBIS axially along the electron beam where they are radially confined by the space charge of the electron beam and longitudinally confined by electrostatic barriers at either end of the trap region within the solenoid. Ions are held in the trap until successive electron impact ionization breeds the desired charge state, and then the ions are ejected towards the downstream accelerator system. The EBIS can produce pulse trains of ions of a specific charge to mass ratio at a rate of $5 \mathrm{~Hz}$, while a change in element or 
charge state requires $1 \mathrm{~s}$ for adjustment of magnetic components in the downstream beam transport line. The charge capacity of the EBIS trap is a function of the electron beam current, electron energy, and the length of the trap according to the equation

$$
C=\frac{I}{e} \times l \times \sqrt{\frac{m_{e}}{2 E}} .
$$

The Extended EBIS upgrade will include the addition of a second superconducting solenoid to effectively double the length available for gas injection, ion transfer, and ion trapping as shown in Fig. 3. A system for ${ }^{3} \mathrm{He}$ polarization and gas injection will be installed in the upstream portion of the new solenoid. A conceptual illustration of the proposed ${ }^{3} \mathrm{He}$ ion source is shown in Fig. 4. The ${ }^{3} \mathrm{He}$ ion source will consist of a glass polarization cell adjacent to a narrow vacuum chamber, which serves as an ionization cell for efficient gas ionization. The polarization and ionization cells will be connected by a high speed pulsed valve designed to operate in a $5 \mathrm{~T}$ magnetic field similar to the valve used to pulse ${ }^{4} \mathrm{He}$ for the RHIC Optically-Pumped Polarized $\mathrm{H}^{-}$Ion Source (OPPIS) [17]. A differential pumping scheme will surround the ionization cell to maintain an ultra-high vacuum in the rest of the EBIS vacuum system. The pressure in the polarization cell will be in the range of 1-3 mbar. However, the amount of ${ }^{3} \mathrm{He}$ injected will be small enough that after injection into the ionization cell, the ${ }^{3} \mathrm{He}$ will quickly expand to pressures below $10^{-6} \mathrm{mbar}$, which is necessary to preserve the electron beam quality and prevent discharges from the electron beam to the ionization cell walls.

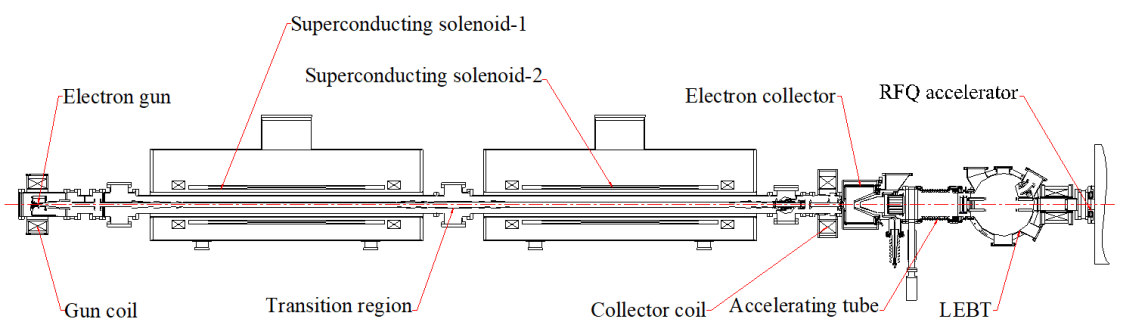

Figure 3: The Extended EBIS will consist of two $5 \mathrm{~T}$ solenoids to increase the ion trap length with additional pumping to improve the vacuum quality. Infrastructure for direct gas injection into the EBIS will be installed in the upstream solenoid.

Excluding the ionization cell, an ultra-high vacuum in the range of $10^{-10}$ mbar is required for the production of high-charge state ions, and residual ${ }^{3} \mathrm{He}$ gas could lower the maximum achievable polarization because ${ }^{3} \mathrm{He}$ in the EBIS vacuum will depolarize over time and can be ionized during later EBIS fills. Therefore, it is necessary to minimize the amount of ${ }^{3} \mathrm{He}$ injected into the EBIS vacuum both to preserve the limited supply of ${ }^{3} \mathrm{He}$ and to maintain the ultra-high vacuum conditions in the EBIS trap. The narrow diameter of the ionization cell reduces the volume that is filled with injected ${ }^{3} \mathrm{He}$, and the constrictions on either end of the ionization cell help confine the ${ }^{3} \mathrm{He}$ in the ionization cell where it is more likely to be ionized by the electron beam. 


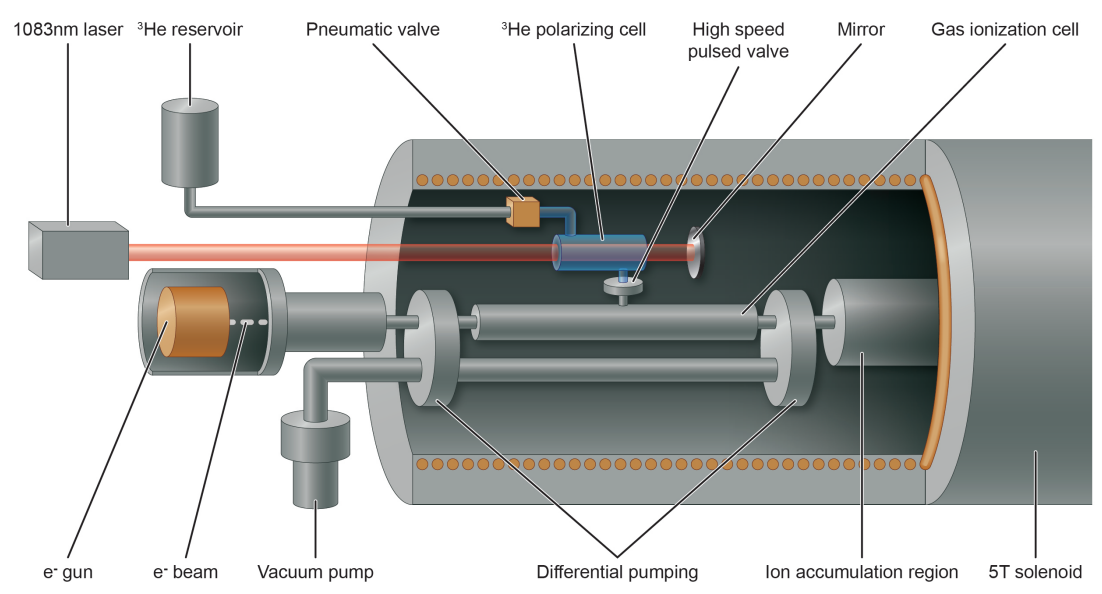

Figure 4: Conceptual illustration of the proposed ${ }^{3} \mathrm{He}$ ion source to be installed in the upgraded RHIC EBIS.

\section{4. ${ }^{3}$ He Injection \& Ionization Simulations}

Expanding on the preliminary results reported in 2017 [12], the entire Extended EBIS vacuum system has been modeled (see Fig. 5) to estimate the vacuum performance of the gas injection system and the efficiency of the gas ionization. In addition to testing the feasibility of gas injection, various design options including the dimensions of the ionization cell are tested to optimize the final design. Since the pressures in the EBIS vacuum will be below $10^{-6} \mathrm{mbar}$, the vacuum is in the molecular flow regime, and MolFlow is used to create the EBIS model [18, 19]. Molflow models consist of several 2-dimensional facets that represent the surfaces of vacuum chambers as well as the locations of vacuum pumps and gas injection.

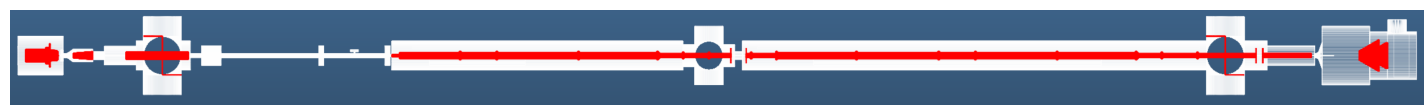

Figure 5: Model of the entire Extended EBIS vacuum structure in MolFlow. Internal structures are colored red for contrast.

The electron beam of the EBIS acts as an ion pump on the EBIS vacuum by ionizing gaseous atoms and trapping them in the beam. Thus the electron beam can be modeled in MolFlow by a set of facets that make a long narrow cylinder with the radius of the electron beam. The facets of the electron beam are semi-transparent pumps with a opacity equal to the probability of a gas atom being ionized while traversing the electron beam. Thus simulated atoms are either ionized and removed from the simulation or traverse the electron beam without any change in their trajectory. The probability of ionization upon hitting the electron beam facets is equal to the ratio of the average distance traveled through the electron beam, which is equal to the diameter of the electron beam, and the mean free path of gas atoms in the electron beam, and when these values are determined in the electron rest frame, the ionization probability per facet hit can be given by

$$
R=\frac{16 I \sigma}{3 \pi^{2} e r_{e} v_{\text {gas }}}
$$




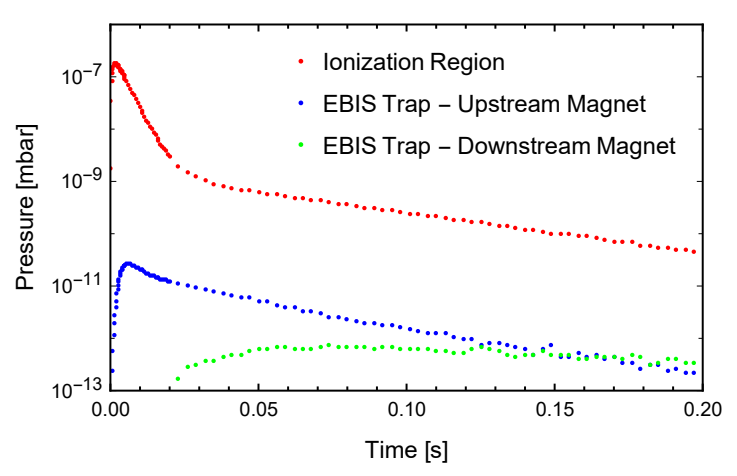

(a) ${ }^{3} \mathrm{He}$ pressure in the EBIS trap.

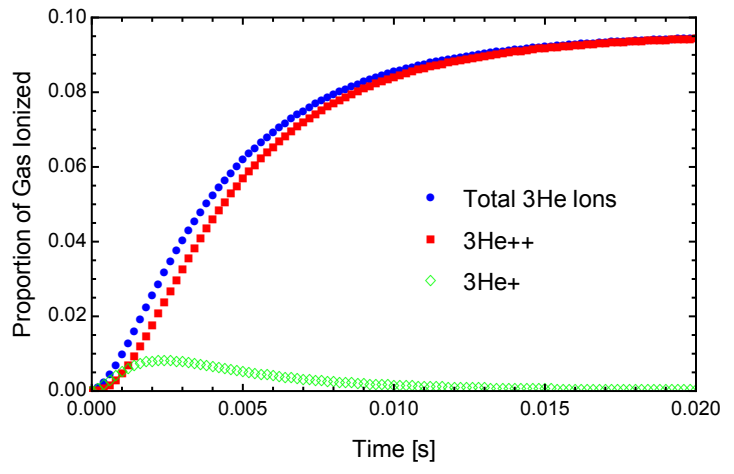

(b) Populations of ${ }^{3} \mathrm{He}^{+}$and ${ }^{3} \mathrm{He}^{++}$ions.

Figure 6: Results from simulations of gas injection into the Extended EBIS.

where $I$ is the electron beam current, $r_{e}$ is the radius of the electron beam in the MolFlow model, $v_{g a s}$ is the average thermal speed of the gas, and $\sigma$ is the electron-impact ionization cross-section, which is determined as a function of electron energy from Eq. 4 in [20] using parameters from $[20,21,22]$. The average gas speed is modified by a factor of $\frac{3 \pi}{8}$ to account for the fact that faster gas molecules will hit the electron beam facets more often in the simulation. This simplified model of the electron beam will accurately simulate a real electron beam with a nonuniform distribution of electrons because the electron beam radius dependence in the ionization probability $R$ cancels with the radius in the model geometry, and thus simulations results are independent of the radius chosen.

Results from the ${ }^{3} \mathrm{He}$ injection and ionization simulations are encouraging for the feasibility of an operational polarized ${ }^{3} \mathrm{He}^{++}$ion source. Fig. 6a shows that the ${ }^{3} \mathrm{He}$ pressure at various locations in the Extended EBIS trap drops to below the $10^{-10}$ mbar level necessary for efficient high-charge state ion production within $200 \mathrm{~ms}$, which is significantly less than the $1 \mathrm{~s}$ timing between switching ion species. Fig. $6 \mathrm{~b}$ shows the proportion of injected ${ }^{3} \mathrm{He}$ atoms ionized into the ${ }^{3} \mathrm{He}^{+}$and ${ }^{3} \mathrm{He}^{++}$charge states by the electron beam for the final ionization cell design chosen for the Extended EBIS upgrade. The actual number of ${ }^{3} \mathrm{He}^{++}$ions produced will depend on the amount of ${ }^{3} \mathrm{He}$ gas injected, which is highly dependent on the pulsed valve design and the ${ }^{3} \mathrm{He}$ pressure in the polarization cell. The operation of the pulsed valve also has to be optimized including timing and number of pulses per fill. However, these ionization results imply that achieving the goal of $2.5 \times 10^{113} \mathrm{He}^{++}$ions per pulse is achievable. Time scales for the production of ${ }^{3} \mathrm{He}^{++}$ions and operation of the EBIS with the final ionization cell design are shown in Table 1, and all of the time results are indicative of a successful design.

\section{Conclusion}

Progress is steadily being made on the development of a polarized ${ }^{3} \mathrm{He}^{++}$ion source for the EIC. Installation of the Extended EBIS upgrade is scheduled for the end of the summer of 2020, and installation of components for polarization and injection of ${ }^{3} \mathrm{He}$ is scheduled for the summer of 2021. In parallel with development of a ${ }^{3} \mathrm{He}^{++}$ion source, a spin-flipper and polarimeter are being developed to test the feasibility of the ion source after the ${ }^{3} \mathrm{He}^{++}$ions have been accelerated to 


\begin{tabular}{|lc|}
\hline Step sequence & Time \\
\hline${ }^{3} \mathrm{He}$ gas injection & $0.5 \mathrm{~ms}$ \\
Diffusion into ionization cell & $2 \mathrm{~ms}$ \\
Injected gas pressure falls $50 \%$ & $5 \mathrm{~ms}$ \\
Ionization of ${ }^{3} \mathrm{He} \mathrm{to}^{3} \mathrm{He}^{+}$ & $\sim 10 \mathrm{~ms}$ per gas injection \\
Time constant for ${ }^{3} \mathrm{He}^{+} \rightarrow{ }^{3} \mathrm{He}^{++}$conversion & $1 \mathrm{~ms}$ \\
Pump down to $10^{-9} \mathrm{mbar}$ & $\sim 30 \mathrm{~ms}$ \\
$5 \mathrm{~Hz}$ EBIS pulse repetition rate & $200 \mathrm{~ms}$ \\
Switching time between species & 1 second \\
\hline
\end{tabular}

Table 1: EBIS polarized ${ }^{3} \mathrm{He}^{++}$ion production with a $10 \mathrm{Amp}, 20 \mathrm{keV}$ electron beam.

$6 \mathrm{MeV}$. Successful measurement of greater than $70 \%$ polarization at $6 \mathrm{MeV}$ will confirm the ability to provide polarized ${ }^{3} \mathrm{He}^{++}$ions to the EIC to study the nuclear spin structure of the neutron.

\section{Acknowledgments}

This research is supported by the Program for R\&D for Next Generation Nuclear Physics Accelerator Facilities of the DOE Office of Nuclear Physics under contract numbers DE-SC0008740 and DE-SC0012704.

\section{References}

[1] W. E. Burcham, O. Karban, S. Oh and W. B. Powell, A source of polarized 3 He ions, Nuclear Instruments and Methods 116 (1974) 1.

[2] D. Findley, S. Baker, E. Carter and N. Stockwell, A polarized 3He+ ion source, Nuclear Instruments and Methods 71 (1969) 125.

[3] R. Slobodrian, C. Rioux, J. Giroux and R. Roy, A polarized 3He ion source for electrostatic accelerators, Nuclear Instruments and Methods in Physics Research Section A: Accelerators, Spectrometers, Detectors and Associated Equipment 244 (1986) 127.

[4] Report of the electron ion collider advisory committee, tech. rep., 2009.

[5] Report of the Community Review of EIC Accelerator R\&D for the Office of Nuclear Physics, tech. rep., DOE Office of Nuclear Physics, 2017.

[6] E. National Academies of Sciences and Medicine, An Assessment of U.S.-Based Electron-Ion Collider Science. The National Academies Press, Washington, DC, 2018, 10.17226/25171.

[7] C. S. Epstein, Development of a polarized 3He ion source for RHIC, AIP Conference Proceedings 1441 (2012) 643.

[8] C. S. Epstein, Development of a Polarized Helium-3 Ion Source for RHIC using the Electron Beam Ion Source, B. S. Thesis, Massachusetts Institute Of Technology, 2013.

[9] J. D. Maxwell, C. S. Epstein and R. G. Milner, Diffusive Transfer of Polarized 3He Gas through Depolarizing Magnetic Gradients, 1412.6167. 
[10] J. D. Maxwell, Development of a Polarized 3He Beam Source for RHIC with EBIS, in PSTP2015, 2015 .

[11] J. Maxwell, C. Epstein, R. Milner, J. Alessi, E. Beebe, A. Pikin et al., Development of a Polarized Helium-3 Source for RHIC and eRHIC, International Journal of Modern Physics: Conference Series 40 (2016) 1660102.

[12] M. Musgrave, R. Milner, G. Atoian, E. Beebe, S. Kondrashev, A. Pikin et al., Polarized 3He++ Ion Source for RHIC and an EIC, PoS PSTP2017 (2018) .

[13] A. Zelenski, G. Atoian, E. Beebe, D. Raparia, J. Ritter, J. Maxwell et al., Optically-Pumped Polarized $H$ - and $3 H e++$ Ion Sources Development at RHIC, PoS SPIN2018 (2018) .

[14] T. R. Gentile, P. J. Nacher, B. Saam and T. G. Walker, Optically polarized He3, Reviews of Modern Physics 89 (2017) [1612.04178].

[15] A. Nikiel-Osuchowska, G. Collier, B. Głowacz, T. Pałasz, Z. Olejniczak, W. P. Wȩglarz et al., Metastability exchange optical pumping of 3 He gas up to hundreds of millibars at 4.7 tesla, European Physical Journal D 67 (2013).

[16] J. Alessi, Electron Beam Ion Source Pre-Injector Project, Tech. Rep. BNL-73700-2005-IR, Brookhaven National Laboratory, 2005.

[17] I. Alekseev, C. Allgower, M. Bai and E. Al., Configuration Manual Polarized Proton Collider at RHIC, tech. rep., Brookhaven National Laboratory, January, 2006.

[18] R. Kersevan and J.-L. Pons, Introduction to MOLFLOW+: New graphical processing unit-based Monte Carlo code for simulating molecular flows and for calculating angular coefficients in the compute unified device architecture environment, Journal of Vacuum Science \& Technology A: Vacuum, Surfaces, and Films 27 (2009) 1017.

[19] M. Ady, Monte Carlo Simulations of Ultra High Vacuum and Synchrotron Radiation for Particle Accelerators, Ph.D. thesis, Ecole Polytechnique, Lausanne, 5, 2016.

[20] W. Lotz, An empirical formula for the electron-impact ionization cross-section, Zeitschrift für Physik 206 (1967) 205.

[21] W. Lotz, Electron-impact ionization cross-sections and ionization rate coefficients for atoms and ions from hydrogen to calcium, Zeitschrift für Physik 216 (1968) 241.

[22] W. Lotz, Electron-impact ionization cross-sections and ionization rate coefficients for atoms and ions from scandium to zinc, Zeitschrift für Physik 220 (1969) 466. 